\title{
Evolución y estado de la investigación en contabilidad ambiental
}

\section{Noelia Araújo Vila, Jose Antonio Fraiz Brea y Lucília Cardoso}

\author{
Departamento de Economía Financiera y Contabilidad, Universidad de Vigo \\ Departamento de Organización de Empresas y Marketing, Universidade de Vigo \\ CITUR , Centro de Investigação, Desenvolvimento e Inovação em Turismo. Leiria-Portugal
}

La contabilidad ambiental es un área que ha mostrado un destacado aumento en investigación, sobre todo a partir de la década de 1990 y especialmente en los últimos diez años. Todos los aspectos relacionados a la sostenibilidad e impacto ambiental son temas de preocupación social actual, por lo que son necesarios planes y políticas para su gestión y control. Vinculada a dichos planes aparece la contabilidad como facilitadora de identificación, registro y control de las acciones previstas para reducir el impacto ambiental. Tras contextualizar el área de estudio de la contabilidad ambiental, este trabajo se propone analizar el estado y evolución de la investigación en esta área, cuantificando las publicaciones y determinando los tópicos de estudio más habituales. Para ello, se realiza una revisión bibliométrica y un análisis de contenido deductivo, además de un análisis de correlación de los temas de investigación más habituales en contabilidad ambiental. Entre las áreas más estudiadas, emerge la sostenibilidad, el desarrollo sostenible, el impacto ambiental o el cambio climático, siempre desde un punto de vista ligado a la contabilidad.

Palabras clave: contabilidad ambiental, revisión bibliométrica, análisis deductivo, correlación

\section{Evolution and status of environmental accounting research}

Environmental accounting is an area of accounting that has shown an outstanding increase in research, especially since the 1990s, and especially in the last 10 years. All aspects related to sustainability and environmental impact are issues of current social concern and plans and policies are needed for its management and control. Linked to these plans, accounting appears as a facilitator of identification, registration and control of the planned actions to reduce the environmental impact. The present work, after contextualizing this area of study, makes an analysis of the state and evolution of the research in this area, quantifying the publications and determining the most common study topics. For this purpose, a bibliometric review and a deductive content analysis is carried out, as well as a correlation analysis of the most common research topics in environmental accounting. As main results, among the studied topics, they stand out sustainability, sustainable development, environmental impact or climate change, always from a point of view linked to accounting.

Keywords: environmental accounting, bibliometric review, deductive analysis, correlation

\section{Evoluçáo e estado da arte em contabilidade ambiental}

A contabilidade ambiental é uma área da contabilidade, que se tem destacado e apresentado um incremento de investigação, sobretudo a partir da década de 1990 e especialmente nos últimos 10 anos. Os aspetos relacionados com a sustentabilidade e impacto ambiental são temas de preocupação atual, sendo necessários planos e politicas para a sua 
gestão e controle. Vinculada a estes planos, surge a contabilidade como facilitadora de identificação, registo e controle das açôes previstas para reduzir o impacto ambiental. Por este facto, o presente trabalho, contextualiza esta área de estudo, realiza uma análise do estado e evolução da investigação deste setor quantificando as publicaçóes e determinando os temas mais correntes. Para o efeito, efetuamos uma revisão bibliométrica e uma análise de conteúdo dedutivo, complementando com uma análise de correlação dos temas de investigação mais correntes em contabilidade ambiental. Entre as áreas mais estudadas, emergem a sustentabilidade, o desenvolvimento sustentável, o impacto ambiental e as alteraçôes climáticas, sempre ligados à contabilidade.

Palavras-chave: contabilidade ambiental, revisão bibliométrica, análise dedutiva, correlação

\section{Introducción}

Las pautas de comportamiento humano y de las organizaciones están muchas veces relacionadas con el origen de diversos problemas ambientales. Hasta el siglo pasado, la sociedad no estaba realmente preocupada por esta situación. Sin embargo, desde finales del siglo XX, se confirma la existencia de un alto nivel de concienciación ambiental. Estudios como los de Scott y Willits (1994) y Dietz, Stern y Guagnano (1998); muestras norteamericanas; Gooch (1995) en un estudio comparativo con muestras de Suecia, Lituania y Letonia, Aragonés y Amérigo (1991); y Corraliza, Berenguer, Muñoz y Martín (1995) en muestras españolas respaldan dicho argumento.

Actualmente, la sostenibilidad y preocupación ambiental están en un punto álgido en cuanto a concientización y difusión. Por una parte, la sociedad muestra preocupación por la situación actual del planeta, como por problemas como el calentamiento global o la contaminación. Por otra parte, son ya muchos los sectores e industrias que incluyen medidas sostenibles para minimizar la actual situación e impacto ambiental.

La sostenibilidad es aplicada en la actualidad en numerosos y diversos campos, como el turismo, la moda, la alimentación o la construcción, entre otros. Es una realidad presente en el día a día y de la que debe haber mayor concientización social. Incluso, en sectores a priori ajenos a esta situación, la sostenibilidad y respeto ambiental están presentes. Uno de ellos es el de la contabilidad y finanzas. Asociamos la contabilidad al registro que surge de las transacciones comerciales presentes en la sociedad. Efectivamente, esta es la raíz de la contabilidad, que se define como «una ciencia social que reúne un conjunto de conocimientos, normas y principios contables, y aplica rigurosamente una metodología que le permite obtener resultados razonables, los cuales son expresados en los estados financieros» (Zevallos, 2016, p. 94). Entre otras definiciones, se menciona que la contabilidad se configura como «la ciencia económica que atiende a la información explicativa, predictiva y de control, de la medida y agregación del valor de la riqueza y de la renta generada en el intercambio de los sujetos privados y públicos» (Ibáñez, 2010, p. 10). En definitiva, se trata de una ciencia social por su contenido de carácter económico (Arévalo \& Quinche, 2010), que tiene el siguiente objetivo:

El conocimiento pasado, presente y futuro de la realidad económica, en términos cuantitativos en todos sus niveles organizativos, mediante métodos específcos, apoyados en bases suficientemente contrastadas, a fin de elaborar información que cubra las necesidades financieras, $y$, las de planificación y control internas (Canibaño, 1996, p. 22). 
La contabilidad ha pasado por varias etapas hasta la consolidación de su propia teoría y criterios normativos. Así, el Comité de Normas Internacionales de Contabilidad (IASC) emitió las Normas Internacionales de Contabilidad (NICs). El IASC dio paso a la Junta de Normas Internacionales de Contabilidad (IASB). Esta ha trabajado y desarrollado las Normas Internacionales de Información Financiera (NIIF) con el fin de garantizar la toma de decisiones de los inversores en todo el mundo. De esta manera, se busca evitar el surgimiento de nuevas y pronunciadas crisis financieras, que afectarían a organizaciones y economías nacionales (Peña, 2013).

En paralelo a la evolución de la práctica de la contabilidad, se ha realizado investigación en este campo. Así, en la década de 1970, surgieron las primeras preocupaciones sobre el estado y desarrollo de la investigación contable, ya que «no existía una teoría completamente aceptada sobre la forma de producir la información contable externa» (Chua, 1986, p. 38). De esto derivó el surgimiento de diferentes teorías para la formulación de políticas, las cuales dieron lugar a la creación de escuelas de contabilidad, aunque sin llegar un consenso (Villarreal \& Córdoba, 2017).

Ya en la década de 1980, otros académicos (Dornbusch \& Fischer, 1980; Hopwood, 1983; Tinker, 1980) intentaron publicar sus trabajos contables en revistas reconocidas bajo un enfoque multidisciplinar como, por ejemplo, en The Accounting Review. Así, se generaron dos nuevas corrientes en el campo de la investigación contable, conocidas como corriente interpretativa y corriente crítica. Antes de su aparición, existía la corriente principal, que estaba más centrada en el funcionamiento de la contabilidad financiera y vinculada con la regulación - normas -, «la cual ha producido importantes resultados en pro del beneficio de los usuarios de la información contable financiera» (Giraldo,
2008, p. 77). A partir de la proliferación de investigaciones, surgieron revistas propias para su difusión como Accounting, Organizations and Society o Critital Perspectives on Accounting (Villarreal \& Martínez, 2017). Previas a ellas, existía ya la corriente principal.

Tras revisar literatura científica vinculada al área de contabilidad, se pueden establecer infinidad de líneas de investigación sobre esta área: la línea contable financiera, la línea contable gubernamental, la línea contable socio-economía, la línea contable de gestión, la línea contable administrativa, la línea contable social, etc. (Araujo \& García, 2008). Entre todas estas líneas, tiene cabida la contabilidad ambiental, pues, en un campo inicialmente ligado al mundo de las transacciones, emerge también la necesidad de llevar registro de las actividades vinculadas a la sostenibilidad y temas ambientales. A la par de la proliferación de la investigación ambiental, emerge la investigación en contabilidad ambiental desde la década de 1990.

Con tres décadas de historia, se trata de un campo relativamente novedoso que surge del movimiento ambiental y sostenible (concienciación ambiental). Ya que se trata de un campo todavía nuevo y desconocido, el presente trabajo pretende profundizar más en su estudio. Asimismo, se busca conocer cuáles son los aspectos más habituales en la investigación de este sector.

Para ello, en primer lugar, el presente trabajo contextualiza el concepto de contabilidad a partir de la revisión literaria del término. En segundo lugar, en la parte empírica del presente trabajo, se busca indagar sobre cuáles son las revistas con más publicaciones sobre el área de contabilidad ambiental y los autores más populares, y cómo ha sido la evolución cuantitativa de la investigación en esta área; asimismo, se realiza un análisis de la última década sobre los temas 
y tópicos de investigación más habituales, y las correlaciones entre los mismos. De esta manera, se busca obtener mayor información sobre la contabilidad ambiental.

\section{Contabilidad ambiental}

El alcance de la contabilidad como ciencia social va más allá de aspectos financieros, económicos y administrativos de la sociedad, es decir, traspasa estimaciones, medidas e información económico-financiera. Actualmente, la contabilidad no gira solo en torno a números y beneficios económicos, sino también en torno al beneficio y bienestar social. Desde ya hace algunas décadas, se considera a la sostenibilidad como parte del bienestar (Pacheco et al., 2017). La sostenibilidad y preservación del medio ante futuras generaciones preocupa cada vez más a la sociedad, sobre todo ante el evidente impacto ambiental. Por eso, se hacen necesarios planes, programas y políticas coordinados entre estados, empresas y población civil. Dichos planes necesitan a su vez sistemas de gestión, por lo que entra en juego «la contabilidad como facilitadora de identificación, registro y control de las acciones planificadas para reducir el impacto ambiental» (Higuera Martínez, Restrepo, Rojas \& Franco, 2017, p. 88).

Según Bortone y Méndez Vergara (2004), la contabilidad ambiental surge como «una herramienta que genera información útil para la toma de decisiones; para ello debe considerar la base conceptual que surge de la relación, hasta hace poco ignorada, de las empresas con el ambiente en el cual interactúan» (p. 7). Esta área de estudio tiene una trayectoria de más de cincuenta años. Hoy en día, argumentos de la década de los 90 siguen siendo vigentes, como que «las ciencias contables deben expandir su campo de acción para incluir los datos sociales y el impacto en el medio ambiente incluyendo la auditoría ambiental»
(Mathews, 1997, p. 494). Precisamente, a inicios de la década de 1990 la contabilidad ambiental presenta su mayor crecimiento respecto de décadas anteriores, pues, en ese momento, gerentes, políticos y la población civil en general toman consciencia de las interacciones de las empresas con el medio ambiente. De esta manera, se hace necesario identificar y valorar dichas interacciones, algo que no había pasado hasta el momento (Mathews, 1997).

Más allá del auge de este campo en los 90, ya en la década de 1970 había surgido investigación al respecto. En ese periodo, aparecen publicaciones sobre contabilidad ambiental de autores como Mobley (1970), Churchman (1971) o Beams y Fertig (1971). En los años 80 y 90, varios expertos trabajaron en la conceptualización del área, como Gray y Maunders (1987); Gray, Bebbington, y Walters (1993); Mathews (1993); y Mathews y Perera (1995) (ver Tabla 1).

A partir de la década del 2000, la sociedad ha tomado incluso mayor consciencia del concepto de sostenibilidad e impacto ambiental, por lo que la investigación en contabilidad ambiental sigue en auge. Aparecen en esa década trabajos sobre auditoría ambiental (Gray, 2000), determinantes de la innovación ambiental en la industria (Brunnermeier \& Cohen, 2003), riesgo de impacto ambiental (Wood, 2003), la necesidad de unidades de contabilidad normalizadas para temas medioambientales (Boyd \& Banzhaf, 2007) o la huella de carbono (Monfreda, Wackernagel \& Deumling, 2004; Wiedmann \& Minx, 2008), entre otros.

La década de 2010 es la que presenta mayor volumen de investigaciones en este campo. De hecho, el 50\% de la totalidad de investigaciones en el área de contabilidad ambiental se han publicado a partir del año 2010. Por ello, se analizará esta década detalladamente en la parte empírica de este trabajo. 
Tabla 1. Definiciones de contabilidad ambiental de las décadas 80 y 90

\begin{tabular}{|c|c|}
\hline Definición & Autores \\
\hline $\begin{array}{l}\text { El proceso de comunicar los efectos sociales y ambientales de las organizaciones económicas a grupos de interés, particularmente } \\
\text { dentro de la sociedad y a la sociedad en general. Como tal, implica extender la responsabilidad de las organizaciones (particu- } \\
\text { larmente las empresas), más allá de la función tradicional de proporcionar una cuenta financiera a los propietarios del capital } \\
\text { y, en particular, a accionistas. Tal extensión se basa en la suposición de que las empresas tienen responsabilidades más amplias } \\
\text { que simplemente hacer dinero para sus accionistas. }\end{array}$ & $\begin{array}{l}\text { Gray y } \\
\text { Maunders } \\
(1987)\end{array}$ \\
\hline $\begin{array}{l}\text { Divulgaciones voluntarias de información, tanto cualitativa como cuantitativa, realizadas por organizaciones para informar } \\
\text { o influenciar una gama de audiencias. Las divulgaciones cuantitativas pueden ser en términos financieros o no financieros. }\end{array}$ & $\begin{array}{l}\text { Mathews } \\
(1993, \text { p. } 64)\end{array}$ \\
\hline $\begin{array}{l}\text { Se puede considerar que cubre todas las áreas de contabilidad que pueden verse afectadas por la responsabilidad empresarial a } \\
\text { problemas ambientales, incluidas nuevas áreas de eco-contabilidad. }\end{array}$ & $\begin{array}{l}\text { Gray et al. } \\
(1993, \text { p. 6) }\end{array}$ \\
\hline $\begin{array}{l}\text { Por lo menos, la contabilidad social significa una extensión de la divulgación en áreas no tradicionales, como proporcionar } \\
\text { información sobre empleados, productos, servicio a la comunidad, y prevención o reducción de la contaminación. Sin embargo, } \\
\text { el término "contabilidad social" también se usa para describir una forma integral de contabilidad que toma en cuenta las } \\
\text { externalidades. Las organizaciones del sector también pueden ser evaluadas de esta manera, aunque la mayoría de los escritores } \\
\text { sobre el tema de la contabilidad social parecen preocuparse por las organizaciones del sector privado. }\end{array}$ & $\begin{array}{l}\text { Mathews y } \\
\text { Perera, (1995, } \\
\text { p. 364). }\end{array}$ \\
\hline
\end{tabular}

Fuente: Elaboración propia sobre la base de Gray y Maunders (1987), Mathews (1993), Gray et al. (1993) y Mathews y Perera (1995).

\section{Metodología}

Los artículos analizados se han recogido de la base de datos Scopus y corresponden al periodo que va desde el 2010 hasta el 2019. Se toman en cuenta los artículos in press. En primer lugar, se han filtrado los datos seleccionando como subárea de estudio «contabilidad» dentro del área de estudio «negocios, dirección y contabilidad». Esto arroja un resultado de un total de 134 revistas vigentes en el año 2017, de las cuales 6 son Open Access (ver Tabla 2).

Como segundo filtro y con el fin de cuantificar los artículos vinculados a contabilidad, se ha usado como palabra clave de búsqueda en el título, palabras clave o abstract de los artículos la palabra "account» (contabilidad). A partir de ello, se ha obtenido un total de 631.951 trabajos (ver Tabla 3). Destaca el año 2017 como el más productivo en este campo, con 82.897 artículos. A continuación, se ha realizado un filtro a través de las palabras «environmental + account», con lo que se ha obtenido un total de 49.783 trabajos, 27.606 correspondientes a la etapa 2010-2019. Como último filtro se han seleccionado los trabajos del área de ciencias sociales, que corresponden a 6.367 en total, mientras que 3.834 corresponden al periodo 2010-2019. Esta sería la muestra final (ver Tabla 3). Dicha muestra será la analizada mediante análisis de contenido deductivo.

Tabla 2. Número de revistas Scopus que publican artícullos del área de contabilidad

\begin{tabular}{|c|c|c|}
\hline año & $\mathbf{n}^{\text {o }}$ revistas & open access \\
\hline 2017 & 134 & 6 \\
\hline 2016 & 131 & 6 \\
\hline 2015 & 131 & 6 \\
\hline 2014 & 131 & 6 \\
\hline 2013 & 130 & 6 \\
\hline 2012 & 130 & 6 \\
\hline 2011 & 131 & 6 \\
\hline
\end{tabular}


Tabla 3. Artícullos Scopus con la palabra account, environmental+account y, finalmente, environmental+account del área de ciencias sociales en su título, palabras clave o abstract (2010-2019)

\begin{tabular}{|c|c|c|c|c|c|c|}
\hline \multirow[b]{2}{*}{ Año } & \multicolumn{2}{|c|}{ Account } & \multicolumn{2}{|c|}{ Environmental + account } & \multicolumn{2}{|c|}{$\begin{array}{l}\text { Environmental+acount } \\
\text { Ciencias sociales }\end{array}$} \\
\hline & Artículos & Porcentaje & Artículos & Porcentaje & Artículos & Porcentaje \\
\hline 2019 & 221 & $3,50 \%$ & 22 & $0,08 \%$ & - & - \\
\hline 2018 & 49.258 & $7,79 \%$ & 2.716 & $9,84 \%$ & 416 & $10,85 \%$ \\
\hline 2017 & 82.897 & $13,12 \%$ & 3.845 & $13,93 \%$ & 587 & $15,31 \%$ \\
\hline 2016 & 78.080 & $12,36 \%$ & 3.628 & $13,14 \%$ & 523 & $13,64 \%$ \\
\hline 2015 & 76.122 & $12,05 \%$ & 3.332 & $12,07 \%$ & 504 & $13,15 \%$ \\
\hline 2014 & 74.670 & $11,82 \%$ & 3.117 & $11,29 \%$ & 409 & $10,67 \%$ \\
\hline 2013 & 72.721 & $11,51 \%$ & 3.129 & $11,33 \%$ & 385 & $10,04 \%$ \\
\hline 2012 & 69.840 & $11,05 \%$ & 2.833 & $10,26 \%$ & 376 & $9,81 \%$ \\
\hline 2011 & 66.214 & $10,48 \%$ & 2.645 & $9,58 \%$ & 335 & $8,74 \%$ \\
\hline 2010 & 61.928 & $9,80 \%$ & 2.339 & $8,47 \%$ & 299 & $7,80 \%$ \\
\hline TOTAL & 631.951 & $100,00 \%$ & 27.606 & $100,00 \%$ & 3.834 & $100,00 \%$ \\
\hline
\end{tabular}

* Datos resaltados en recuadro son los valores máximos de cada serie

El análisis de los datos sigue, por lo tanto, dos métodos: 1) análisis bibliométrico, para el cual se han considerado las siguientes variables: títulos de los artículos, palabras clave y abstract; y 2) análisis de contenido deductivo aplicado a palabras clave de los artículos y fuentes de los trabajos (revistas), y análisis de correlación de palabras clave. El proceso de análisis incluyó dos pasos principales: la elaboración de una matriz de codificación y la codificación de los datos en las categorías de la matriz. Las palabras se adoptaron como la unidad de registro y la frecuencia fue la regla de enumeración principal para la codificación. En el proceso de análisis se utilizó el programa QSR NVivo 11. Finalmente, se realizó un análisis de coeficiente de correlación intraclase (CCI) a través del estadístico $\rho$ de Pearson. Dicho coeficiente se usa como la probabilidad de establecer una ecuación lineal entre dos variables; por cada cambio de unidad en una de ellas se espera un cambio de unidad (correlativo) en la otra, sin tener en cuenta ni la magnitud ni la escala de medición de las variables (Zhou, Deng, Xia \& Fu, 2016). El CCI existe para cuantificar la concordancia entre diferentes mediciones de una variable numérica y extiende su uso al caso en el que se dispone de más de dos observaciones por sujeto. Es un indicador de la fiabilidad de una sola medida determinado por la siguiente expresión:

$$
\rho=\frac{\operatorname{Var}(\pi)}{\operatorname{Var}(\pi)+\operatorname{Var}(\varepsilon)}
$$

Los valores del CCI pueden oscilar entre 0 y 1 , de modo que la máxima concordancia posible corresponde a un valor de $\mathrm{CCI}=1$. Por otro lado, el valor $\mathrm{CCI}=0$ se obtiene cuando la concordancia observada es igual a la que se espera que ocurra solo por azar. 


\section{Resultados}

En primer lugar, se han analizado las revistas sobre contabilidad presentes en Scopus (total de la población). Se ha establecido un ranking del Top Ten (las diez mejores) sobre la base de su "citeScore» (su impacto o puntuación). Las tres primeras del ranking, Journal of Finances, Journal of Financial Economics y Review of Financial Studies, son también aquellas cuyos artículos son los más citados, con cifras que superan las 1400 citas. Con respecto a este aspecto, Journal of Financial Economics es la revista cuyos trabajos han sido los más citados, con un total de 2002 citas (ver Tabla 4). En cuanto a editoriales, Elsevier es la que posee mayor número de revistas en el Top Ten, con un total de cinco.

A continuación, se analizan los autores, afiliaciones y países con más trabajos en el total de publicaciones del área de contabilidad, así como las palabras clave más repetidas, antes de pasar a analizar la muestra seleccionada (ver Tabla 5). En cuanto a los autores, los más productivos presentan más de 100 trabajos, entre los que destaca Anon con 631 investigaciones. Las afiliaciones con mayor número de trabajos publicados son el Centre National de la Recherche Scientifique (CNRS), la Russian Academy of Sciences y la Chinese Academy of Sciences, cada una de ellas con más de 11.000 trabajos publicados desde el año 2010. El país que se encuentra a la cabeza con respecto a las publicaciones en el área de contabilidad es Estados Unidos, con más del doble de publicaciones respecto del siguiente en el ranking, Reino Unido. Les siguen Francia y Alemania, cada uno de ellos con más de 109.000 trabajos publicados.

Para obtener más información de la investigación ligada a la contabilidad, resulta de interés conocer cuáles son las palabras clave más utilizadas. Tal y como se muestra en la Figura 1, la misma palabra «article» es la más habitual, seguida de «human»y «bumans»,

Tabla 4. Ranking Top Ten de mejores revistas en el área contabilidad, base Scopus

\begin{tabular}{|c|c|c|c|c|c|c|c|}
\hline \# & Revistas & $\begin{array}{l}\text { Cite } \\
\text { score }\end{array}$ & percentil & $\begin{array}{l}\text { Recuento } \\
\text { de citas }\end{array}$ & $\begin{array}{l}\text { Resultados } \\
\text { académicos }\end{array}$ & SJR & Editorial \\
\hline 1 & Journal of Finance & 6,81 & 99 & 1540 & 226 & 18,318 & Wiley-Blackwell \\
\hline 2 & Journal of Financial Economics & 5,79 & 98 & 2002 & 346 & 12,489 & Elsevier \\
\hline 3 & Review of Financial Studies & 5,31 & 98 & 1456 & 274 & 14,237 & Oxford University Press \\
\hline 4 & Management Accounting Research & 4,53 & 97 & 299 & 66 & 1,426 & Elsevier \\
\hline 5 & Journal of Accounting and Economics & 4,36 & 96 & 510 & 117 & 6,875 & Elsevier \\
\hline 6 & Accounting, Auditing and Accountability Journal & 4,33 & 95 & 676 & 156 & 2,187 & Emerald \\
\hline 7 & Journal of Accounting Research & 4,29 & 95 & 425 & 99 & 6,957 & Wiley-Blackwell \\
\hline 8 & British Accounting Review & 3,31 & 94 & 268 & 81 & 0,986 & Elsevier \\
\hline 9 & Accounting Review & 3,24 & 93 & 796 & 246 & 3,946 & $\begin{array}{l}\text { American Accounting } \\
\text { Association }\end{array}$ \\
\hline 10 & Critical Perspectives on Accounting & 3,18 & 92 & 560 & 176 & 1,773 & Elsevier \\
\hline
\end{tabular}


«priority journal», y «female» $\mathrm{y}$ «male». Dichos datos no aportan información relevante de los subcampos de investigación dentro del área de contabilidad, sino de las palabras que se creen prioritarias a la hora de describir el trabajo, como que se trata de un artículo, de investigación vinculada a humanos o de la técnica estadística usada en el mismo.
Centrándonos en el área del objeto de estudio, la contabilidad ambiental, el total de trabajos publicados en Scopus es de 66.990, de los cuales el primero se remonta a 1914. Como se muestra en el Figura 2, los últimos años son los más prolíferos. Entre 2004 y la actualidad, se han publicado el $75 \%$ de los trabajos del total; y, a partir del 2011, el 50\%.

Tabla 5. Ranking de autores, afiliaciones y países con mayores publicaciones en Scopus

\begin{tabular}{|c|c|c|c|c|c|}
\hline Autores & No trabajos & Afiliación & No trabajos & País & No trabajos \\
\hline Anon & 631 & CNRS Centre National de la Recherche Scientifique & 23.250 & Estados Unidos & 383.505 \\
\hline Shukla, P.K. & 129 & Russian Academy of Sciences & 14.369 & Reino Unido & 141.256 \\
\hline Hayat, $T$. & 121 & Chinese Academy of Sciences & 11.770 & Francia & 112.726 \\
\hline Sodha, M.S. & 118 & University of Oxford & 8.166 & Alemania & 109.636 \\
\hline Schober, $R$. & 117 & University of Cambridge & 8.090 & China & 95.855 \\
\hline Faessler, $A$. & 115 & UCL & 7.799 & Italia & 75.342 \\
\hline Sapountzakis, E.J. & 115 & University of Toronto & 7.370 & Canadá & 61.679 \\
\hline Guedes Soares, C. & 110 & University of California, Berkeley & 7.344 & Japón & 56.511 \\
\hline Ruckenstein, E. & 109 & Inserm & 7.228 & Rusia & 53.660 \\
\hline Guiochon, G. & 108 & University of Tokyo & 6.598 & España & 51.872 \\
\hline
\end{tabular}

Fuente: Elaboración propia.

Figura 1. Palabras clave más habituales en la investigación en el área de contabilidad

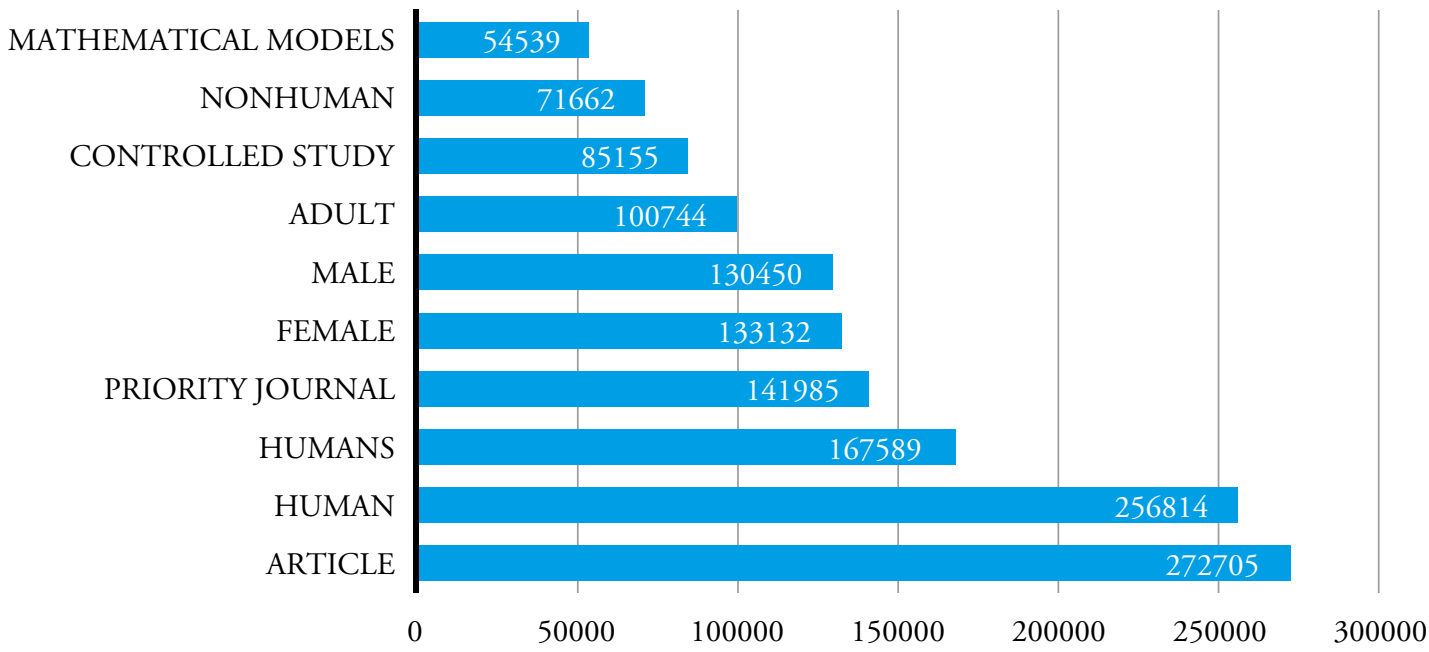


Figura 2. Evolución de publicaciones de contabilidad ambiental (1914-2019)

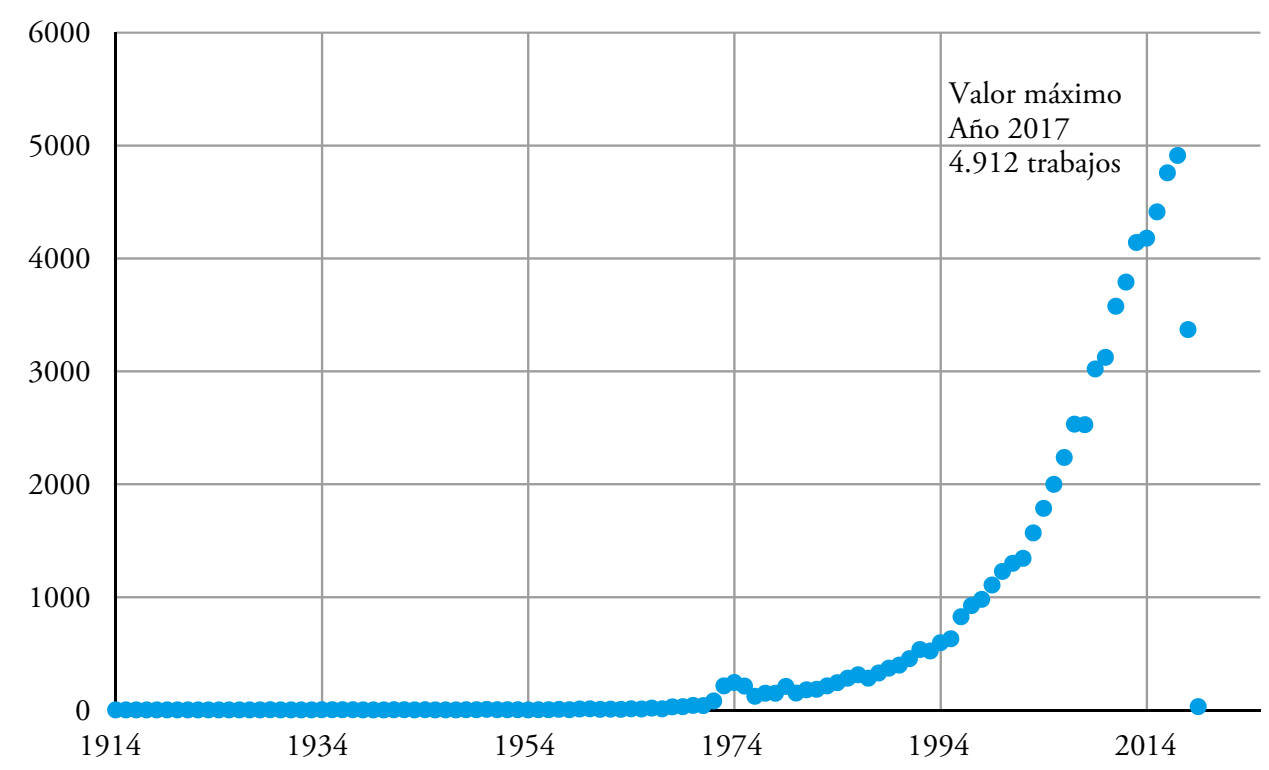

$\mathrm{Al}$ analizar la muestra seleccionada (3.834 trabajos), siguen destacando la Chinese Academy of Sciences y la CNRS como las dos primeras afiliaciones en esta área. Emerge en tercer lugar la Arizona State University, universidad que no aparecía en el Top Ten del ranking general. Le siguen la University of Oxford y el Wageningen University and Research Centre. En cuanto a la presencia geográfica, de nuevo destacan Estados Unidos (864 trabajos), Reino Unido (482), China (243), Australia (238) e Italia (220). El primer país de habla hispana que aparece en el ranking es España, con el sexto lugar con 214 trabajos de contabilidad ambiental publicados en el periodo analizado.

En cuanto a los autores más destacados, se encuentran Wood con siete artículos, y Beaver, Macharis y Stadler con seis cada uno (ver Tabla 6). Tras revisar los títulos y abstracts de los trabajos del Top Ten de autores (un total de 54 trabajos), se establecen como temas principales de estudio el impacto ambiental, la sostenibilidad, el transporte y la polución (ver Tabla 6).

Tras analizar el total de palabras clave de la muestra, se establece un ranking con las veinte palabras con mayores frecuencias. Así, «sostenibilidad», «desarrollo sostenible» e «impacto ambiental» se posicionan como los tópicos más usados en la contabilidad ambiental. Un análisis más detallado por año muestra que en 2018 los tres primeros puestos del ranking corresponden a estos mismos tópicos. En cambio, entre 2010 y 2011, la palabra clave más usada era "artículo». Ya en el 2012, "desarrollo sostenible» emerge como líder; y, a partir, de 2015, «sostenibilidad» (ver Tabla 7).

$\mathrm{Al}$ repetirse el análisis de frecuencias con las fuentes o revistas de investigación ( $\mathrm{N}=1.747)$, se establece como líder en publicaciones del área ambiental Sustainability Switzerland, con 128 trabajos en el periodo analizado (ver Tabla 8). 
Tabla 6. Autores con mayor número de publicaciones en contabilidad ambiental

\begin{tabular}{|c|c|c|c|}
\hline Autor & No trabajos & Referencias & Temas \\
\hline Wood, R. & 7 & $\begin{array}{l}\text { Stadler et al. (2018); Wood et al. (2018); Tukker et al. } \\
\text { (2018); Hamilton et al. (2018); Ivanova et al. (2016); } \\
\text { Steen-Olsen et al. (2016); Wood et al. (2016) }\end{array}$ & $\begin{array}{l}\text { Tablas input/output ambientales; impacto } \\
\text { ambiental en el comercio; productos no alimen- } \\
\text { tarios; impacto ambiental; consumo hogares; } \\
\text { huella carbono; consumo doméstico }\end{array}$ \\
\hline $\begin{array}{l}\text { Beaver, } \\
\text { K.M. }\end{array}$ & 6 & $\begin{array}{l}\text { Beaver et al. (2015); Schwartz \& Beaver (2014); Maynard } \\
\text { et al. (2014); Beaver et al. (2013); Beaver et al. (2011); } \\
\text { Beaver (2011) }\end{array}$ & $\begin{array}{l}\text { Entorno genético; criminología; factores } \\
\text { ambientales; genética y ambiente; biosocial; } \\
\text { moderadores ambientales }\end{array}$ \\
\hline $\begin{array}{l}\text { Macharis, } \\
\text { C. }\end{array}$ & 6 & $\begin{array}{l}\text { Messagie (2015); Van Lier \& Macharis (2014); Van Lier } \\
\text { et al. (2014); Macharis et al. (2014); Hollevoet et al. } \\
\text { (2011); Macharis et al. (2010) }\end{array}$ & $\begin{array}{l}\text { Desempeńo ambiental de vehículos; impacto } \\
\text { ambiental del transporte; teletrabajo; sostenibili- } \\
\text { dad; transporte sostenible; movilidad; logística }\end{array}$ \\
\hline $\begin{array}{l}\text { Stadler, } \\
\text { K. }\end{array}$ & 6 & $\begin{array}{l}\text { Stadler et al. (2018); Wood et al. (2018); Tukker et al. } \\
\text { (2018); Hamilton et al. (2018); Ivanova et al. (2016); } \\
\text { Wood et al. (2015) }\end{array}$ & $\begin{array}{l}\text { Tablas input/output ambientales; impacto } \\
\text { ambiental; comercio; sostenibilidad }\end{array}$ \\
\hline $\begin{array}{l}\text { Armah, } \\
\text { F.A. }\end{array}$ & 5 & $\begin{array}{l}\text { Kangmennaang Osei et al. (2016); Armah et al. (2016); } \\
\text { Yengoh \& Armah (2016); Armah et al. (2013); Armah } \\
\text { et al. (2013) }\end{array}$ & $\begin{array}{l}\text { Genética; condiciones de trabajo; disparidad; } \\
\text { polución }\end{array}$ \\
\hline Jordan, A. & 5 & $\begin{array}{l}\text { Benson et al. (2013); Jordan \& Turnpenny (2013); Bauer } \\
\text { et al. (2013); Benson et al. (2012); Berkhout et al. (2010) }\end{array}$ & $\begin{array}{l}\text { Dirección ambiental; colaboración; políticas } \\
\text { públicas; escenarios y ejercicios de política }\end{array}$ \\
\hline $\begin{array}{l}\text { Tukker, } \\
\text { A. }\end{array}$ & 5 & $\begin{array}{l}\text { Stadler et al. (2018); Wood et al. (2018); Tukker et al. } \\
\text { (2018); Ivanova et al. (2016); Wood et al. (2015) }\end{array}$ & $\begin{array}{l}\text { Tablas input/output ambientales; impacto } \\
\text { ambiental en el comercio; productos no alimen- } \\
\text { tarios; impacto ambiental; sostenibilidad global }\end{array}$ \\
\hline $\begin{array}{l}\text { Verburg, } \\
\text { P.H. }\end{array}$ & 5 & $\begin{array}{l}\text { Malek et al. (2018); Scherer et al. (2018); Zagaria et al. } \\
\text { (2017); Verburg (2014); Andrés et al. (2012) }\end{array}$ & $\begin{array}{l}\text { Cambio global; sostenibilidad; agricultura; } \\
\text { medio ambiente; biodiversidad }\end{array}$ \\
\hline Yepes, V. & 5 & $\begin{array}{l}\text { Navarro et al. (2018); Penadés-Plà, et al. (2018); Sierra } \\
\text { et al. (2017); Penadés-Plà et al. (2017); Sierra et al. (2017) }\end{array}$ & Ciclo de vida social; sostenibilidad social \\
\hline $\begin{array}{l}\text { Giljum, } \\
\text { S. }\end{array}$ & 4 & $\begin{array}{l}\text { Stadler et al. (2018); Wood et al. (2018); Tukker et al. } \\
\text { (2018); Wood et al. (2015). }\end{array}$ & $\begin{array}{l}\text { Tablas input/output ambientales; comercio; } \\
\text { impacto ambiental: sostenibilidad global }\end{array}$ \\
\hline
\end{tabular}

Tabla 7. Top 20 frecuencias palabras clave $(\mathrm{N}=8.810)$

\begin{tabular}{|c|c|c|c|c|c|c|c|c|c|c|c|}
\hline Ranking & Palabra clave & 2018 & 2017 & 2016 & 2015 & 2014 & 2013 & 2012 & 2011 & 2010 & TOTAL \\
\hline $1^{\circ}$ & Sostenibilidad & 48 & 79 & 57 & 66 & 34 & 36 & 27 & 28 & 19 & 328 \\
\hline $2^{\circ}$ & Desarrollo sostenible & 43 & 71 & 55 & 54 & 43 & 26 & 35 & 32 & 25 & 319 \\
\hline $3^{\circ}$ & Impacto ambiental & 34 & 51 & 43 & 37 & 22 & 25 & 18 & 18 & 22 & 242 \\
\hline $4^{\circ}$ & Cambio climático & 33 & 56 & 54 & 37 & 35 & 45 & 25 & 35 & 29 & 216 \\
\hline $5^{\circ}$ & Humano & 29 & 80 & 51 & 50 & 52 & 35 & 31 & 31 & 26 & 207 \\
\hline $6^{\circ}$ & Tomar decisiones & 23 & 36 & 32 & 33 & 25 & 17 & 15 & 18 & 20 & 162 \\
\hline
\end{tabular}




\begin{tabular}{|c|c|c|c|c|c|c|c|c|c|c|c|}
\hline Ranking & Palabra clave & 2018 & 2017 & 2016 & 2015 & 2014 & 2013 & 2012 & 2011 & 2010 & TOTAL \\
\hline $7^{\circ}$ & Humanos & 13 & 56 & 36 & 45 & 46 & 31 & 26 & 25 & 22 & 158 \\
\hline $8^{\circ}$ & Estados Unidos & 12 & 34 & 28 & 30 & 29 & 24 & 28 & 21 & 23 & 155 \\
\hline $9^{\circ}$ & Artículo & 18 & 30 & 22 & 26 & 28 & 41 & 34 & 39 & 31 & 139 \\
\hline $10^{\circ}$ & China & 29 & 38 & 28 & 22 & 24 & 15 & 10 & 9 & 9 & 129 \\
\hline $11^{\circ}$ & Política ambiental & 27 & 43 & 27 & 27 & 30 & 31 & 26 & 17 & 21 & 125 \\
\hline $12^{\circ}$ & Ambiental & 11 & 22 & 20 & 24 & 18 & 14 & 13 & 13 & 9 & 122 \\
\hline $13^{\circ}$ & Protección ambiental & 18 & 27 & 39 & 23 & 24 & 10 & 17 & 19 & 19 & 115 \\
\hline $14^{\circ}$ & Dirección ambiental & 15 & 26 & 21 & 23 & 15 & 15 & 16 & 13 & 12 & 110 \\
\hline $15^{\circ}$ & Economía ambiental & 23 & 41 & 25 & 18 & 22 & 25 & 15 & 11 & 15 & 107 \\
\hline $16^{\circ}$ & Mujer & 8 & 31 & 16 & 23 & 21 & 14 & 12 & 12 & 13 & 99 \\
\hline $17^{\circ}$ & Hombre & 9 & 31 & 17 & 22 & 19 & 14 & 11 & 9 & 12 & 99 \\
\hline $18^{\circ}$ & Evaluación de riesgos & 14 & 28 & 24 & 18 & 19 & 15 & 16 & 16 & 10 & 94 \\
\hline $19^{\circ}$ & Evaluación del impacto ambiental & 10 & 22 & 20 & 9 & 7 & 14 & 12 & 12 & 7 & 85 \\
\hline $20^{\circ}$ & Modelo numérico & 10 & 10 & 14 & 8 & 15 & 9 & 17 & 10 & 12 & 84 \\
\hline \multicolumn{2}{|c|}{ Palabra más repetida rank. } & $1^{\circ}$ & $5^{\circ}$ & $1^{\mathrm{o}}$ & $1^{\mathrm{o}}$ & $5^{\circ}$ & $4^{\circ}$ & $2^{\circ}$ & $9^{\circ}$ & $9^{\circ}$ & \\
\hline
\end{tabular}

Fuente: Elaboración propia.

Tabla 8. Top 20 fuentes de los trabajos $(\mathrm{N}=1.747)$

\begin{tabular}{|c|l|c|c|l|c|}
\hline Rank & \multicolumn{1}{|c|}{ Revista } & Frecuencia & Rank & \multicolumn{1}{|c|}{ Revista } & Frecuencia \\
\hline $1^{\circ}$ & Sustainability Switzerland & 128 & $11^{\circ}$ & Global Environmental Change & 27 \\
\hline $2^{\circ}$ & Land Use Policy & 66 & $12^{\circ}$ & Marine Policy & 24 \\
\hline $3^{\circ}$ & Building And Environment & 48 & $13^{\circ}$ & Ecosystem Services & 23 \\
\hline $4^{\circ}$ & Journal Of Industrial Ecology & 47 & $14^{\circ}$ & Journal Of Flood Risk Management & 22 \\
\hline $5^{\circ}$ & $\begin{array}{l}\text { Transportation Research Part D Transport } \\
\text { And Environment }\end{array}$ & 46 & $15^{\circ}$ & $\begin{array}{l}\text { Journal Of Environmental Planning And } \\
\text { Management }\end{array}$ \\
\hline $6^{\circ}$ & Environmental Science And Policy & 41 & $16^{\circ}$ & Applied Geography & 20 \\
\hline $7^{\mathrm{o}}$ & Water Switzerland & 32 & $17^{\circ}$ & Environment Development And Sustainability & 20 \\
\hline $8^{\circ}$ & Journal Of Ecology And Rural Environment & 30 & $18^{\circ}$ & Social Science And Medicine & 20 \\
\hline $9^{\circ}$ & Environmental Impact Assessment Review & 28 & $19^{\circ}$ & Transportation Research Procedia & 20 \\
\hline $10^{\circ}$ & Sustainable Cities And Society & 28 & $20^{\circ}$ & $\begin{array}{l}\text { International Archives Of The Photogrammetry } \\
\text { Remote Sensing And Spatial Information Sciences } \\
\text { ISPRS Archives }\end{array}$ & 19 \\
\hline
\end{tabular}


Finalmente, una vez establecidos los tópicos más estudiados en dicha área, se buscan posibles correlaciones entre los mismos o asociaciones temáticas, operación ya realizada en investigaciones previas de otras áreas de la contabilidad (Porte, Saur-Amaral \& Pinho, 2018). Tras aplicar el estadístico $\rho$ de Pearson, se buscan los resultados más próximos a 1, pues estas son las correlaciones más fuertes. En concreto, los trabajos de dirección ambiental tratan a su vez la sostenibilidad y el desarrollo sostenible. Los de sostenibilidad tratan también de desarrollo sostenible, impacto ambiental, toma de decisiones y dirección ambiental. El desarrollo sostenible se vincula con sostenibilidad, toma de decisiones y dirección ambiental. La evaluación de riesgos está altamente vinculada a los trabajos sobre humanos, economía ambiental y política ambiental. Las únicas variables que no muestran relaciones aceptables con el resto son el modelo numérico, con valores próximos a 0 en todos los casos; artículo, con débil correlación con variables como cambio climático, humanos o evaluación de impacto ambiental. Los países más investigados en esta área, Estados Unidos y China, no tienen relación entre sí (ver Tabla 9).

Tabla 9. Correlaciones entre las temáticas más habituales investigadas en la contabilidad ambiental

\begin{tabular}{|c|c|c|c|c|c|c|c|c|c|c|c|c|c|c|c|c|c|c|c|c|}
\hline & $S$ & $D S$ & $I A$ & $C C$ & $H$ & $T D$ & $H S$ & $E U$ & $A R$ & $\mathrm{CH}$ & $P O A$ & $A M$ & PRAM & $D A$ & $E A$ & $M$ & HO & $E R$ & $E I A$ & $M N$ \\
\hline$S$ & 1,00 & 0,93 & 0,94 & 0,74 & 0,82 & 0,90 & 0,66 & 0,43 & $-0,45$ & 0,86 & 71 & ,82 & & 0,95 & 0,76 &, 72 &, 80 &, 80 &, 63 & $-0,41$ \\
\hline$D S$ & 93 & 00 & ,89 & 0,67 & 0,89 & 0,92 & 0,72 & 0,54 & & & & & & & & & & & & \\
\hline$I A$ & 94 & 0,89 & 1,00 & 0,80 & 0,77 & 0,90 & 0,54 & 0,35 & $-0,54$ & $-0,86$ & 0,69 & 0,68 & & 0,88 & 0,81 & 0,63 & 0,74 & 0,77 & 0,70 & $-0,34$ \\
\hline$C C$ & 0,74 & 0,67 & 0,80 & 1,00 & 0,76 & 0,69 & 0,61 & 0,44 & $-0,11$ & 0,70 & 0,65 & 0,63 & & 0,70 & & & & & 0,85 & $-0,29$ \\
\hline$H$ & 0,82 & ,89 & 0,77 &, 76 & 1,00 & 0,73 & 0,52 & 0,75 & $-0,20$ & 0,79 & & & & & & & & & ,63 & $-0,10$ \\
\hline$T D$ & 0,90 & 92 & 0,90 &, 69 & 0,73 & 1,00 & 0,73 & 0,52 & $-0,57$ & 0,83 & & & &, 88 &, 65 & & 83 &, 77 &, 48 & $-0,27$ \\
\hline$H S$ & 0,66 & 0,72 & 0,54 & 0,61 & 0,52 & 0,73 & 1,00 & 0,89 & 0,01 & 0,55 & 0,70 & & & 0,76 & & & 0,94 & 0,79 & 0,42 & $-0,06$ \\
\hline$E U$ & 0,43 & 0,54 & 0,35 & 0,44 & 0,75 & 0,52 & 0,89 & 1,00 & 0,18 & 0,25 & 0,54 & 0,74 & ,43 & 0,67 & 0,41 &, 83 & 0,80 &, 67 &, 40 & 0,21 \\
\hline$A R$ & $-0,45$ & $-0,52$ & $-0,54$ & $-0,11$ & $-0,20$ & $-0,57$ & 0,01 & 0,18 & 1,00 & $-0,64$ & $-0,14$ & $-0,24$ & & $-0,33$ & $-0,23$ & & $-0,15$ & $-0,21$ & 0,00 & $-0,07$ \\
\hline $\mathrm{CH}$ & 0,86 & 0,88 & $-0,86$ & 0,70 & 0,79 & 0,83 & 0,55 & 0,25 & $-0,64$ & 1,00 & 0,78 & & & 0,75 & 0,86 & & 0,71 & 0,77 & 0,55 & $-0,18$ \\
\hline$P O A$ & 0,71 & 0,70 & 0,69 & 0,65 & 0,83 & 0,58 & 070 & 0,54 & $-0,14$ & 0,78 & 1,00 & 0,57 & 20 & 0,72 & 0,95 & 0,75 &, 82 & 0,72 &, 58 & $-0,10$ \\
\hline$A M$ & 0,82 & & 0,68 & & & 0,85 & 0,88 & 0,74 & $-0,24$ & 0,63 & & & & & & & 0,84 & 0,80 & 0,45 & $-0,18$ \\
\hline PRAM & 0,53 & 0,70 & 0,63 & 0,57 & 0,58 & 0,75 & 0,45 & 0,43 & $-0,58$ & 0,56 & & & & 0,58 & 0,34 & 0,41 & 0,46 & 0,70 & 0,50 & 0,26 \\
\hline$D A$ & 0,95 & 0,93 & 0,88 & & & & & 0,67 & $-0,33$ & 0,75 & 0,72 & & &, 00 & 0,70 & & 0,86 & 0,85 & 0,67 & $-0,22$ \\
\hline$E A$ & 0,76 & & 0,81 & & & & & 0,41 & $-0,23$ & 0,86 & & & & & & & & & 0,71 & $-0,19$ \\
\hline$M$ & 0,72 & 0,77 & 0,63 & 0,59 & 0,94 & 0,78 & 0,97 & 0,83 & $-0,04$ & 0,61 & 0,75 & & 0,41 & 0,80 & 0,68 & & 0,98 & 0,76 & 0,42 & $-0,18$ \\
\hline $\mathrm{HO}$ & 0,80 & 0,82 & 0,74 & 0,67 & 0,96 & 0,83 & 0,94 & 0,80 & $-0,15$ & 0,71 & 0,82 & 0,84 & 0,46 & 0,86 & 0,78 & 0,98 & 1,00 & 0,80 & 0,50 & $-0,19$ \\
\hline$E R$ & 0,80 & 0,89 & 0,77 & 0,82 & 0,92 & 0,77 & 0,79 & 0,67 & $-0,21$ & 0,77 & 0,72 & 0,80 & 0,70 & 0,85 & 0,76 & 0,76 & 0,80 & 1,00 & 0,81 & 0,04 \\
\hline & 0,63 & & & & & & & 0,40 & 0,00 & & & & & & & & & & 1,00 & $-0,06$ \\
\hline$M N$ & $-0,41$ & $-0,12$ & $-0,34$ & $-0,29$ & $-0,10$ & $-0,27$ & $-0,06$ & 0,21 & $-0,07$ & $-0,18$ & $-0,10$ & $-0,18$ & 0,26 & $-0,22$ & $-0,19$ & $-0,18$ & $-0,19$ & 0,04 & $-0,06$ & 1,00 \\
\hline
\end{tabular}

S: sostenibilidad; DS: desarrollo sostenible; IA: impacto ambiental; CC: cambio climático; H: humano; TD: tomar decisiones; HS: humanos; EU: Estados Unidos; AR: artículo; CH: China; POA: política ambiental; AM: ambiental; PARA: protección ambiental; DA: dirección ambiental; EA: economía ambiental; M: mujer; HO: hombre; ER: evaluación de riesgos; EIA: evaluación impacto ambiental; MN: modelo numérico 


\section{Conclusiones}

La investigación en contabilidad abarca un amplio número de áreas de estudio. Asimismo, se trata de una ciencia social que va más allá de números, medidas y estimaciones. Ha evolucionado y se ha adaptado a temas sociales de interés, los cuales también requieren de herramientas y mecanismos de identificación, registro y control. Es por ello que la contabilidad es útil y necesaria en muchos campos. Uno de ellos es el ámbito ambiental, y todo lo vinculado con la sostenibilidad, desarrollo sostenible e impacto ambiental. En pleno siglo XXI, son cada vez más los organismos e individuos que muestran conciencia frente al problema. Temas como el cambio climático, la huella de carbono, la sostenibilidad, entre otros, siguen siendo vigentes, pues generan debate, y se buscan medidas que minimicen o incluso eliminen el impacto ambiental. Además, ya que prácticamente todos los sectores e industrias afectan de algún modo al medio ambiente, considerar la sostenibilidad en todos estos ámbitos se hace necesario para preservar los recursos para el futuro.

Paralelamente al surgir de la consciencia sobre la sostenibilidad, emerge la contabilidad ambiental como herramienta valiosa en este campo. No se trata de una moda pasajera, ya que la investigación en este campo ha pasado de contar con algunos pocos estudios en la primera década del siglo XX a crecer progresivamente desde la década de 1970 . Así, desde la década de 1990, ha habido un salto significativo en la cantidad de investigaciones de este campo y ya en el siglo XXI ha llegado a su máximo apogeo. Además, se ha pasado de publicar los hallazgos de investigaciones de esta área en revistas de contabilidad, a la creación de un gran número de revistas específicas de contabilidad ambiental. Esto muestra el impacto de esta área y la proliferación de trabajos respectivos. Del mismo modo, existen autores que se han centrado en la investigación del campo de la contabilidad ambiental, cada uno con más de cinco publicaciones en la última década. Por el apogeo y amplitud que ha adquirido el campo, han aparecido líneas específicas de análisis, siempre desde el punto de vista contable: entre las más estudiadas en la última década, se encuentran impacto ambiental, sostenibilidad, y transporte o polución.

Por otro lado, a partir del análisis realizado en este trabajo, se han obtenido los veinte términos más usados entre las palabras clave de la muestra estudiada de un total de 8.810 palabras clave de las 3.834 publicaciones científicas publicadas desde 2010. Dichos datos muestran información relevante del estado de la investigación en cuanto a temas más recurrentes y que, por lo tanto, son de mayor interés. Los temas que aparecen con mayor frecuencia son sostenibilidad, desarrollo sostenible, impacto ambiental y cambio climático, lo que los convierte en los principales ítems abordados desde el campo de la contabilidad. Otro dato que llama la atención es que los países que lideran las listas de publicaciones son Estados Unidos y China, pues son las regiones del mundo en las que más investigaciones se realizan sobre el tema. Asimismo, cabe comentar que las investigaciones no muestran resultados aislados sobre un aspecto concreto, sino que existen correlaciones entre los términos. Las investigaciones en contabilidad ambiental se centran en ciertos aspectos, como cambio climático, desarrollo sostenible, evaluación del impacto ambiental, entre otros. A su vez, enmarcan estos aspectos en temas que los abarcan, como la sostenibilidad, que se encuentra vinculada con prácticamente todos los ítems analizados; o tratan más de un aspecto en el mismo trabajo. También se debe mencionar, que, ya que son trabajos de contabilidad, es habitual explicar el modelo numérico de análisis usado, por lo que se entiende que «modelo numérico» se encuentre entre las palabras clave más usadas. 
En definitiva, la contabilidad ambiental se constituye como un área de estudio con fuerte presencia en el campo de la investigación, con un crecimiento cuantitativo destacado. Esto se relaciona con el interés que la consciencia ambiental despierta socialmente.

\section{Referencias bibliográficas}

Andres, S. M., Mir, L. C., Van den Bergh, J. C., Ring, I. \& Verburg, P. H. (2012). Ineffective biodiversity policy due to five rebound effects. Ecosystem Services, 1(1), 101-110. https://doi.org/10.1016/j. ecoser.2012.07.003

Aragonés, J. I. \& Amérigo, M. (1991). Un estudio empírico sobre las actitudes ambientales. Revista de Psicología Social, 6(2), 223-240. https://doi.org/10.1080/02134 748.1991 .10821647

Araujo, J. \& García, J. (2008). Lineas de investigación en contabilidad. Informe final del grupo de investigación contabilidad y conocimiento. Medellín: Universidad Autónoma Latinoamericana de Medellín.

Arévalo, N. \& Quinche, F. (2010). Desarrollo del estudio de caso en contabilidad. Memorias del Foro Virtual de Contabilidad Ambiental y Social. Facultad de Ciencias Económicas de la Universidad de Buenos Aires.

Armah, F. A., Boamah, S. A., Quansah, R., Obiri, S. \& Luginaah, I. (2016). Working conditions of male and female artisanal and small-scale goldminers in Ghana: Examining existing disparities. The Extractive Industries and Society, 3(2), 464-474. https://doi. org/10.1016/j.exis.2015.12.010

Armah, F. A., Luginaah, I. \& Odoi, J. (2013). Artisanal small-scale mining and mercury pollution in Ghana: a critical examination of a messy minerals and gold mining policy. Journal of Environmental Studies and Sciences, 3(4), 381-390. https://doi.org/10.1007/ s13412-013-0147-7

Armah, F. A., Luginaah, I. N., Taabazuing, J. \& Odoi, J. O. (2013). Artisanal gold mining and surface water pollution in Ghana: have the foreign invaders come to stay? Environmental Justice, 6(3), 94-102. https://doi. org/10.1089/env.2013.0006

Bauer, M. W., Green-Pedersen, C., Héritier, A. \& Jordan, A. (Eds.). (2012). Dismantling Public Policy: Preferences, Strategies, and Effects. Oxford: Oxford University Press. https://doi.org/10.1093/acprof:oso/9780199656646. 001.0001

Beams, F. A. \& Fertig, P. E. (1971). Pollution control through social cost conversion. Journal of Accountancy, 1(41), 37-42.

Beaver, K. M. (2011). Environmental moderators of genetic influences on adolescent delinquent involvement and victimization. Journal of Adolescent Research, 26(1), 84-114.https://doi.org/10.1177/0743558410384736

Beaver, K. M., Connolly, E. J., Schwartz, J. A., Al-Ghamdi, M. S. \& Kobeisy, A. N. (2013). Genetic and environmental contributions to stability and change in levels of self-control. Journal of Criminal Justice, 41(5), 300-308. https://doi.org/10.1016/j.jcrimjus.2013. 07.003

Beaver, K. M., Gibson, C. L., Turner, M. G., DeLisi, M., Vaughn, M. G. \& Holand, A. (2011). Stability of delinquent peer associations: A biosocial test of Warr's sticky-friends hypothesis. Crime \& Delinquency, 57(6), 907-927.https://doi.org/10.1177/0011128709332660

Beaver, K. M., Schwartz, J. A. \& Gajos, J. M. (2015). A Review of the Genetic and Gene-Environment Interplay Contributors to Antisocial Phenotypes. In J. Morizot \& L. Kazemian (Eds.), The Development of Criminal and Antisocial Behaviour (pp. 109-122). New York: Springer. https://doi.org/10.1007/978-3319-08720-7_8

Benson, D., Jordan, A. \& Huitema, D. (2012). Involving the public in catchment management: an analysis of the scope for learning lessons from abroad. Environmental Policy and Governance, 22(1), 42-54. https:// doi.org/10.1002/eet.593

Contabilidad y Negocios (14) 28, 2019 / ISSN 1992-1896 
Benson, D., Jordan, A. \& Smith, L. (2013). Is environmental management really more collaborative? A comparative analysis of putative 'paradigm shifts' in Europe, Australia, and the United States. Environment and Planning A, 45(7), 1695-1712. https://doi.org/10.1068/a45378

Berkout, F., Haug, C., Hildingsson, R., Stripple, J. \& Jordan, A. J. (2010). Exploring the future: the role of scenarios and policy exercises. Cambridge: Cambridge University Press.

Bortone, N. \& Méndez Vergara, E. (2004). Contabilidad y ambiente. Una disciplina y un campo para el conocimiento y la acción. Actualidad Contable FACES, 7(8), 7-22.

Boyd, J. \& Banzhaf, S. (2007). What are ecosystem services? The need for standardized environmental accounting units. Ecological Economics, 63(2-3), 616-626. https:// doi.org/10.1016/j.ecolecon.2007.01.002

Brunnermeier, S. B. \& Cohen, M. A. (2003). Determinants of environmental innovation in US manufacturing industries. Journal of Environmental Economics and Management, 45(2), 278-293. https://doi.org/10.1016/ S0095-0696(02)00058-X

Canibaño, L. (setiembre, 1996). Los programas de investigación en contabilidad. Contaduria Universidad de Antioquia, (29) 13-61.

Chua, Wai Fong (1986). Desarrollos radicales en el pensamiento contable. En. M. Gómez \& C. M. Ospina (Eds.), Avances interdisciplinarios para una comprensión crítica de la contabilidad. Textos paradigmáticos de las corrientes heterodoxas. Medellín: Editora L. Vieco e Hijas.

Churchman, C. W. (1971). On the facility, felicity, and morality of measuring social change. The Accounting Review, 46(1), 30-35.

Corraliza, J. A., Santiago, J. B., Cáceres, M. D. M. \& Herreros, R. M. (1995). Perfil de las creencias y actitudes ambientales de la población española. En E. Garrido \& M. C. Herrero Alonso (Coords.), Psicología politica, juridica y ambiental (pp. 327-336). Salamanca: EUDEMA
Dietz, T., Stern, P. C. \& Guagnano, G. A. (1998). Social structural and social psychological bases of environmental concern. Environment and Behavior, 30(4), 450-471. https://doi.org/10.1177/001391659803000402

Dornbusch, R. \& Fischer, S. (1980). Exchange rates and the current account. The American Economic Review, 70(5), 960-971.

Giraldo G. (2008). Repercusión de la teoría crítica en la investigación contable. En D. A. Buenaventura (Dir.), Perspectivas criticas de la contabilidad. "Reflexiones y criticas alternas al pensamiento único". Memorias del VII Simposio Nacional de Investigación Contable y Docencia (pp. 77-94). Bogotá: Universidad Nacional de Colombia, Centro Colombiano de Investigaciones Contables Regional Cundinamarca.

Gooch, G. D. (1995). Environmental beliefs and attitudes in Sweden and the Baltic states. Environment and Behavior, 27(4), 513-539. https://doi.org/10.1177/ 0013916595274004

Gray, R. (2000). Current developments and trends in social and environmental auditing, reporting and attestation: a review and comment. International Journal of Auditing, 4(3), 247-268. https://doi.org/10.1111/10991123.00316

Gray, R., Bebbington, J. \& Walters, D. (1993). Accounting for the Environment, Londres: ACCA.

Gray, R., Owen, D. \& Maunders, K. (1987). Corporate Social Reporting: Accounting and Accountability. Englewood Cliffs, NJ; London: Prentice-Hall International.

Hamilton, H. A., Ivanova, D., Stadler, K., Merciai, S., Schmidt, J., van Zelm, R. \& Wood, R. (2018). Trade and the role of non-food commodities for global eutrophication. Nature Sustainability, 1(6), 314. https://doi. org/10.1038/s41893-018-0079-z

Higuera, V. H., Martínez, G. A. P., Restrepo, S. L., Rojas, O. C. \& Franco, R. A. G. (2017). Contabilidad ambiental, tendencias investigativas mundiales. Producción+ Limpia, 12(1), 88-96. https://doi.org/10.22507/pml. v12n1a9 
Hollevoet, J., De Witte, A. \& Macharis, C. (2011). Improving insight in modal choice determinants: an approach towards more sustainable transport. Urban Transport XVII: Urban Transport and the Environment in the 21st Century, (116), 129. https://doi.org/ 10.2495/UT110121

Hopwood, A. G. (1983). On trying to study accounting in the contexts in which it operates. Accounting Organization and Society, 8(2-3), 287-305 https://doi.org/ $10.1016 / 0361-3682(83) 90035-1$

Ibañez, A. (2010). El estatus epistemológico de la contabilidad. Contabilidad y Decisiones, 2(2), 1-15.

Ivanova, D., Stadler, K., Steen-Olsen, K., Wood, R., Vita, G., Tukker, A. \& Hertwich, E. G. (2016). Environmental impact assessment of household consumption. Journal of Industrial Ecology, 20(3), 526-536. https:// doi.org/10.1111/jiec. 12371

Jordan, A. \& Turnpenny, J. (2012). From dismantling by default to symbolic dismantling: water policy in the United-Kingdom. In M. W. Bauer, A. Jordan, C. Green-Pedersen \& A. Héritier (Eds.), Dismantling Public Policy (pp. 176-199). Oxford: Oxford University Press. https://doi.org/10.1093/acprof:oso/978019965 6646.003 .0008

Kangmennaang, J., Osei, L., Armah, F. A. \& Luginaah, I. (2016). Genetically modified organisms and the age of (Un) reason? A critical examination of the rhetoric in the GMO public policy debates in Ghana. Futures, (83), 37-49. https://doi.org/10.1016/j.futures.2016.03.002

Macharis, C., De Witte, A. \& Turcksin, L. (2010). The Multi-Actor Multi-Criteria Analysis (MAMCA) application in the Flemish long-term decision making process on mobility and logistics. Transport Policy, 17(5), 303-311. https://doi.org/10.1016/j.tranpol.2010.02.004

Macharis, C., Milan, L. \& Verlinde, S. (2014). A stakeholder-based multicriteria evaluation framework for city distribution. Research in Transportation Business \& Management, (11), 75-84. https://doi.org/10.1016/j. rtbm.2014.06.004
Malek, Ž., Verburg, P. H., Geijzendorffer, I. R., Bondeau, A. \& Cramer, W. (2018). Global change effects on land management in the Mediterranean region. Global Environmental Change, (50), 238-254. https:// doi.org/10.1016/j.gloenvcha.2018.04.007

Mathews, M. R. (1993). Socially Responsible Accounting. New York; Londres: Chapman \& Hall.

Mathews, M. R. (1997). Twenty-five years of social and environmental accounting research: is there a silver jubilee to celebrate? Accounting, Auditing \& Accountability Journal, 10(4), 481-531. https://doi.org/10.1108/ EUM0000000004417

Mathews, M.R. \& Perera, M.H.B. (1995). Accounting Theory and Development. $3^{\text {a }}$ ed. Melbourne: Thomas Nelson Australia.

Maynard, B. R., Beaver, K. M., Vaughn, M. G., DeLisi, M. \& Roberts, G. (2014). Toward a bioecological model of school engagement: A biometric analysis of gene and environmental factors. Social Work Research, 38(3), 164-176. https://doi.org/10.1093/swr/svu018

Messagie, M., Sergeant, N., Rangaraju, S., San Felix Forner, J., Oliveira, L., Coosemans, T. \& Van Mierlo, J. (2015). A valuation of the environmental performance of vehicles: an analysis and comparison of two methodologies. Transportation Planning and Technology, 38(3), 335-346. https://doi.org/10.1080/0308106 0.2015 .1008797

Mobley, S. C. (1970). The challenges of socio-economic accounting. The Accounting Review, 45(4), 762-768.

Monfreda, C., Wackernagel, M. \& Deumling, D. (2004). Establishing national natural capital accounts based on detailed ecological footprint and biological capacity assessments. Land Use Policy, 21(3), 231-246. https://doi.org/10.1016/j.landusepol.2003.10.009

Navarro, I. J., Yepes, V. \& Martí, J. V. (2018). Social life cycle assessment of concrete bridge decks exposed to aggressive environments. Environmental Impact Assessment Review, (72), 50-63. https://doi.org/10.1016/j.eiar.2018.05.003 
Pacheco Martínez, G. A., Higuera-Ojito, V. H., Ossa, B., Paola, J., Aguirre Espinosa, J. E. \& González Franco, R. A. (2017). Paradigmas emergentes de la contabilidad y las tendencias investigativas de alto impacto. Revista Lasallista de Investigación, 14(1), 103-111. https://doi.org/10.22507/rli.v14n1a9

Peña Molina, C. H. (2013). Análisis de las diferencias entre las Normas Internacionales de Información Financiera (NIIF) y los Principios de Contabilidad (VEN-NIIF). Revista del Centro de Investigación. Universidad La Salle, 10(39). 135-149.

Penadés-Plà, V., García-Segura, T., Martí, J. V. \& Yepes, V. (2018). An optimization-LCA of a prestressed concrete precast bridge. Sustainability, 10(3), 685. https:// doi.org/10.3390/su10030685

Penadés-Plà, V., Martí, J. V., García-Segura, T. \& Yepes, V. (2017). Life-cycle assessment: A comparison between two optimal post-tensioned concrete box-girder road bridges. Sustainability, 9(10), 1864. https://doi.org/ $10.3390 /$ su9101864

Porte, M., Saur-Amaral, I. \& Pinho, C. (2018). Pesquisa em auditoria: principais temas. Revista Contabilidade \& Finanças, 29(76), 41-59. https://doi.org/10.1590/1808$057 \times 201804410$

Ryan, B., Scapens, R. W., Theobald, M. \& Ganzinelli, C. (2004). Metodología de la investigación en finanzas $y$ contabilidad. Barcelona: Deusto.

Scherer, L. A., Verburg, P. H. \& Schulp, C. J. E. (2018). Opportunities for sustainable intensification in European agriculture. Global Environmental Change, (48), 43-55. https://doi.org/10.1016/j.gloenvcha.2017. 11.009

Schwartz, J. A. \& Beaver, K. M. (2014). A biosocial analysis of the sources of missing data in criminological research. Journal of Criminal Justice, 42(6), 452-461. https://doi.org/10.1016/j.jcrimjus.2014.07.002

Scott, D. \& Willits, F. K. (1994). Environmental attitudes and behaviour: A Pennsylvania survey. Environment and Behaviour, 26(2), 239-260. https://doi.org/ $10.1177 / 001391659402600206$

Sierra, L. A., Pellicer, E. \& Yepes, V. (2017). Method for estimating the social sustainability of infrastructure projects. Environmental Impact Assessment Review, (65), 41-53. https://doi.org/10.1016/j.eiar.2017.02.004

Sierra, L. A., Yepes, V. \& Pellicer, E. (2017). Assessing the social sustainability contribution of an infrastructure project under conditions of uncertainty. Environmental Impact Assessment Review, (67), 61-72. https://doi. org/10.1016/j.eiar.2017.08.003

Stadler, K., Wood, R., Bulavskaya, T., Södersten, C. J., Simas, M., Schmidt, S. \& Giljum, S. (2018). EXIOBASE 3: Developing a time series of detailed environmentally extended multi-regional input-output tables. Journal of Industrial Ecology, 22(3), 502-515. https://doi. org/10.1111/jiec. 12715

Steen-Olsen, K., Wood, R. \& Hertwich, E. G. (2016). The carbon footprint of Norwegian household consumption 1999-2012. Journal of Industrial Ecology, 20(3), 582-592. https://doi.org/10.1111/jiec.12405

Tinker, A. M. (1980). Towards a political economy of accounting: an empirical illustration of the Cambridge controversies. Accounting, Organizations and Society, 5(1), 147-160. https://doi.org/10.1016/03613682(80)90031-8

Tukker, A., de Koning, A., Owen, A., Lutter, S., Bruckner, M., Giljum, S. \& Hoekstra, R. (2018). Towards robust, authoritative assessments of environmental impacts embodied in trade: Current state and recommendations. Journal of Industrial Ecology, 22(3), 585-598. https://doi.org/10.1111/jiec.12716

Van Lier, T. \& Macharis, C. (2014). Assessing the environmental impact of inland waterway transport using a life-cycle assessment approach: The case of Flanders. Research in Transportation Business \& Management, (12), 29-40. https://doi.org/10.1016/ j.rtbm.2014.08.003 
Van Lier, T., de Witte, A. \& Macharis, C. (2014). How worthwhile is teleworking from a sustainable mobility perspective? The case of Brussels capital region. European Journal of Transport and Infrastructure Research, 14(3), 244-267.

Verburg, P. H. (2014). The representation of humanenvironment interactions in land change research and modelling. In M. J. Manfredo, J. J. Vaske, A. Rechkemmer \& E. A. Duke (Eds.), Understanding Society and Natural Resources (pp. 161-177). New York: Springer Dordrecht. https://doi.org/10.1007/978-94017-8959-2_8

Villarreal, J. L. \& Martínez, J. X. C. (2017). Fundamentos para el estudio de las corrientes de investigación en contabilidad. Tendencias, 18(2), 139-151. https://doi. org $/ 10.22267 /$ rtend. 171802.81

Wiedmann, T. \& Minx, J. (2008). A definition of 'carbon footprint'. Ecological Economics Research Trends, (1), $1-11$.

Wood, C. (2003). Environmental Impact Assessment: A Comparative Review. Londres: Pearson Education.

Wood, R., Stadler, K., Bulavskaya, T., Lutter, S., Giljum, S., de Koning, A. \& Simas, M. (2014). Global sustainability accounting-developing EXIOBASE for multi-regional footprint analysis. Sustainability, 7(1), 138-163. https://doi.org/10.3390/su7010138

Wood, R., Stadler, K., Simas, M., Bulavskaya, T., Giljum, S., Lutter, S. \& Tukker, A. (2018). Growth in environmental footprints and environmental impacts embodied in trade: Resource efficiency indicators from EXIOBASE3. Journal of Industrial Ecology, 22(3), 553-564. https://doi.org/10.1111/jiec. 12735
Wood, S. A., Karp, D. S., DeClerck, F., Kremen, C., Naeem, S. \& Palm, C. A. (2015). Functional traits in agriculture: agrobiodiversity and ecosystem services. Trends in Ecology \& Evolution, 30(9), 531-539. https://doi.org/10.1016/j.tree.2015.06.013

Yengoh, G. T. \& Armah, F. A. (2016). Land access constraints for communities affected by large-scale land acquisition in Southern Sierra Leone. GeoJournal, 81(1), 103-122. https://doi.org/10.1007/s10708-0149606-2

Zagaria, C., Schulp, C. J., Kizos, T., Gounaridis, D. \& Verburg, P. H. (2017). Cultural landscapes and behavioural transformations: An agent-based model for the simulation and discussion of alternative landscape futures in East Lesvos, Greece. Land Use Policy, 65, 26-44. https://doi.org/10.1016/j.landusepol.2017.03.022

Zevallos, E. E. C. (2016). Construcción de la teoría contable a partir del análisis de casos. Quipukamayoc, 24(45), 93-97. https://doi.org/10.15381/quipu.v24i 45.12468

Zhou, H., Deng, Z., Xia, Y. \& Fu, M. (2016). A new sampling method in particle filter based on Pearson correlation coefficient. Neurocomputing, (216), 208215. https://doi.org/10.1016/j.neucom.2016.07.036

Fecha de recepción: 15 de Junio de 2018 Fecha de aceptación: 11 de octubre de 2019 Correspondencia: naraujo@uvigo.es jafraiz@uvigo.es lucyalves.lucilia@gmail.com 